\title{
Usability and User-Centered Design - User Evaluation. Experience in Self-Checkout Technologies
}

\author{
Barreto-Fernandes, Francisco Antonio ${ }^{a}$ \& Hernandis-Ortuño, Bernabéb \\ ${ }^{a}$ Associated Professor. LIDA/ ESAD.CR - Instituto Politécnico de Leiria, Portugal. \\ francisco.fernandes@gmail.com \\ ${ }^{b}$ Full Professor. Universitat Politècnica de València. Spain. bhernand@upv.es
}

\section{Resumo}

O crescente avanço das novas tecnologias aplicadas no mercado do retalho, tornam comum a venda de produtos sem o contacto pessoal entre vendedor e comprador, sendo o registo e pagamento efetuado em equipamentos eletrónicos de autoatendimento - "self-checkout”. A utilização em grande escala destes equipamentos obriga o consumidor a participar no processo de atendimento, que anteriormente era feito através da interação com os colaboradores da empresa. O utilizador do sistema self-checkout realiza assim todos os passos da compra, desde pesar os produtos, registá-los e efetuar o pagamento. Este é visto como empregado parcial, cuja participação ou desempenho na prestação de serviços pode ser usado pela empresa para melhorar a qualidade de suas operações (KELLEY, et al 1993). No entanto esta participação nem sempre satisfaz o utilizador, podendo causar experiências negativas relacionadas com falhas de usabilidade. O objetivo deste artigo é apresentar os resultados da avaliação feita pelos utilizadores do sistema self-checkout. Os dados foram recolhidos em Portugal através de questionário realizado presencialmente, resultando em 400 respostas validadas. O estudo analisa o grau de satisfação relativamente à qualidade e usabilidade do sistema, o grau de motivação para a sua adoção, bem como o perfil dos utilizadores. A análise dos dados da amostra revela que os utilizadores têm formação escolar média/alta e utilizam com muita regularidade novas tecnologias. Além disso, apresentam um domínio elevado do sistema e uma fácil aprendizagem da sua utilização. O motivo para utilizar as caixas self-service em vez das tradicionais deve-se principalmente às filas de espera nas caixas com operador e ao pequeno volume de produtos. Na generalidade, a amostra revela um grau elevado de 
satisfação com o serviço e com a qualidade, no entanto, em termos comparativos, as caixas de self-checkout não são consideradas melhores que os caixas tradicionais.

A avaliação da interação com o self-checkout foi classificada segundo vinte e seis atributos do sistema. A análise identifica quatro grupos com caraterísticas similares, dos quais dois apresentam avaliação baixa. A "anulação de artigos registados”, a "pesquisa de artigos sem código de barras", o "registo manual", a "área de ensacamento", as "mensagens de erro", o "sensor de peso" e o "pedido de fatura” são sete atributos críticos do sistema.

Os resultados indicam que, a análise da usabilidade orientada para o serviço self-checkout pode ser determinante para a interação utilizador-sistema. As implicações decorrentes das constatações empíricas são discutidas juntamente com orientações para futuras pesquisas.

Palavras chave: Design de Interação, Autoatendimento, Self-checkout, Avaliação do Utilizador, Usabilidade.

\section{Abstract}

The increasing advance of the new technologies applied in the retail market, make it common to sell products without the personal contact between seller and buyer, being the registration and payment of the products made in electronic equipment of self-checkout. The large-scale use of these devices forces the consumer to participate in the service process, which was previously done through interaction with the company's employees. The user of the self-checkout system thus performs all the steps of the purchase, from weighing the products, registering them and making the payment. This is seen as a partial employee, whose participation or performance in providing services can be used by the company to improve the quality of its operations (KELLEY, et al 1993). However this participation does not always satisfy the user, and may cause negative experiences related to usability failures. This article presents the results of the evaluation by the users of the self-checkout system. The data were collected in Portugal through a questionnaire to 400 users. The study analyzes the degree of satisfaction regarding the quality and usability of the system, the degree of motivation for its adoption, as well as the profile of the users. Analysis of the sample data reveals that users have 
basic or higher education and use new technologies very often. They also have a high domain of the system and an easy learning of its use. The reason for using self-checkout instead of the traditional checkout is mainly due to "queues at checkout with operator" and "at the small volume of products". In general, the sample reveals a high degree of satisfaction with the service and with quality, however, in comparative terms, self-checkout is not considered better than operator checkout. The evaluation of the interaction with the selfcheckout was classified according to twenty-six attributes of the system. The analysis identifies four groups with similar characteristics, of which two have low scores. "Cancellation of registered articles", "search for articles without a bar code", "manual registration", "bagging area", "error messages", "weight sensor" and "invoice request "are seven critical attributes of the system. The results indicate that the usability analysis oriented to the selfcheckout service can be determinant for the user-system interaction. The implications of empirical findings are discussed together with guidelines for future research.

Keywords: Interaction Design, Self service, Self-checkout, User evaluation, Usability.

\section{Introdução}

O desenvolvimento da tecnologia, para a economia digital e o aparecimento de novos competidores colocou em causa o habitual modelo de distribuição e retalho, apresentando desafios a um setor que há uns anos era tipicamente tradicional. Pressionados pela conjuntura económica e pela concorrência, as soluções tecnológicas podem servir para que as empresas evoluam e se diferenciem da concorrência, de forma a permanecerem competitivas nos mercados globalizados.

A constante procura na redução de custos, de aumento de valor e produtividade, faz com que a economia de serviços aposte cada vez mais nas tecnologias self-service permitindo que o trabalho assalariado seja substituído pelo trabalho não remunerado dos consumidores (Glazer 1993). Assim como a produção industrial, também a expansão do self-service parece depender em grande parte da interação bem sucedida entre clientes, máquinas e 
software. Essa união está a fazer no setor dos serviços o que a produção em massa já fez no fabrico (Ritzer 1999).

No desenvolvimento de novos formatos das tecnologias de self-service aplicadas ao retalho, os sistemas de registo e pagamento self-checkout tornaram-se numa escolha muito comum nas grandes superfícies comerciais. A rápida evolução deste tipo de tecnologias alteram significativamente a forma de como os consumidores interagem com os logistas e como os logistas comunicam com os seus clientes. Enquanto que a motivação dos logistas está relacionada com a redução de custos, eficiência, flexibilidade, produtividade e melhoria de desempenho coorporativo (Lee 2009), para muitos clientes, o motivo da utilização do selfservice baseia-se nos benefícios percebidos para realizar a sua própria transação. Segundo Dabholkar, Bobbitt e Lee (2003) e ainda Collier \& Kimes (2013), um dos principais benefícios observados pelos clientes é a de que o self-service permite que os clientes tenham maior eficiência na transação.

Com os equipamentos self-checkout em grandes superfícies comerciais, os clientes querem concluir rapidamente a sua experiência de registo e pagamento de modo a estarem prontos para deixar a loja com as suas compras o mais rápido possível. Estes equipamentos eletrónicos devem ser projetados de forma a facilitar a interação com o utilizador, permitindo completar a tarefa com o menor número de ações possíveis, pois todos os atrasos na experiência de interação são vistos como negativos. Por outro lado, a dificuldade sentida durante as primeiras utilizações, pode causar no utilizador inexperiente a sensação de surpresa, confusão, dificuldade, erro e frustração. Uma experiência negativa poderá levar ao abandono da utilização o serviço. Segundo Johnson (1984) e Dabholkar (1994) existe um processo de avaliação afetivo na formação de expectativas da qualidade do serviço e na disposição para usar as tecnologias self-service.

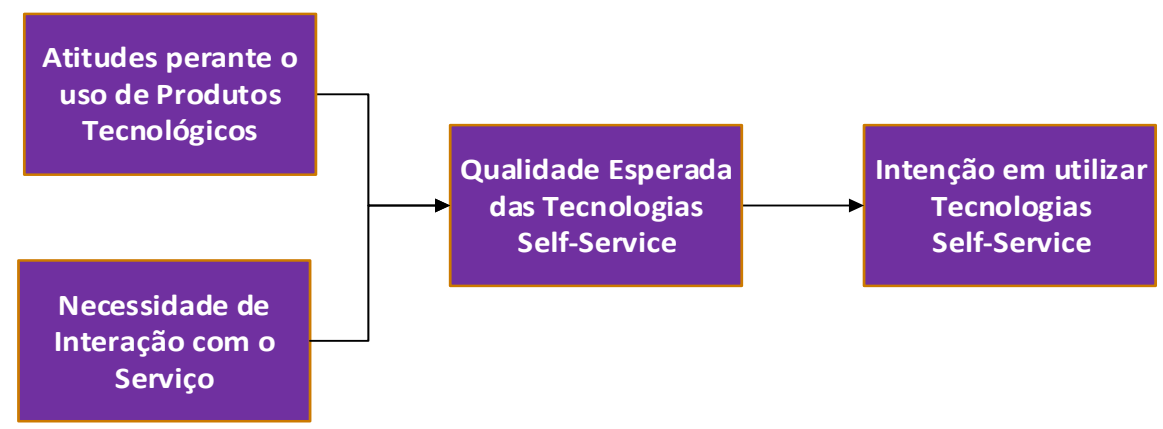

Fig. 1. Processo de avaliação afetivo baseado na disposição para usar Tecnologias Self-service (Dabholkar, 1996). 
Segundo este modelo (Fig.1), aos potenciais consumidores confronta-se a necessidade de interagir com o serviço e a sua disposição para usar produtos tecnológicos. Este modelo indica que o utilizador com atitudes mais positivas perante o uso de produtos tecnológicos tem uma maior predisposição para a utilização da tecnologia, neste caso de um self-service, dado que tem de fazer um menor esforço no momento de tomada de decisão (Bettman e Park, 1980).

Segundo Litfin e Wolfram (2010), a aceitação do cliente de novos produtos e sistemas é crucial para o seu sucesso, dado que a sua implementação falhará se o cliente não os usar e preferir os checkots tradicionais com operador.

\section{Sistemas de registo e pagamento self-checkout}

Os equipamentos self-checkout são uma das aplicações mais difundidas da tecnologia selfservice. A implementação destes sistemas oferecem aos clientes uma alternativa aos checkouts com operador.

Estes equipamentos podem ser constituidos pela combinação de vários módulos, que, conjugados e configurados podem responder de forma mais eficiente às necessidades dos logistas. Por outro lado, estas configurações poderão ser modificadas ao longo do tempo, à medida que as necessidades operacionais se alteram.

Os módulos de auto-pagamento em numerário encontram-se integrados no checkout. Os de auto-pagamento com cartão são efetuados num terminal eletrónico PIN Pad pertencente a uma entidade bancária. Estes são independentes e estão apensos ao corpo do equipamento.

Das diferentes configurações possíveis, a unidade principal é composta por diversos sistemas eletrónicos integrados, podem incorporar diferentes sistemas embebidos e utilizar recursos a tecnologias de informação. Estas tecnologias apresentam-se ao utilizador como um conjunto composto por diferentes periféricos (fig. 2):

- $\quad$ suporte para colocação do cesto/produtos, antes do registo

- monitor LCD Touchscreen

- $\quad$ saída de audio

- leitor de código de barras biótico incorporado na balança de entrada

- leitor de código de barras manual

- suporte dos sacos com os artigos registados, acoplado com a balança saída

- entradas para notas e para moedas

- $\quad$ saídas de trocos: notas e moedas

- $\quad$ saída de recibos

- PIN Pad para efetuar transação bancária eletrónica de débito ou crédito 
- entrada de cartão de débito ou crédito

- impressora de faturas e recibos

- balança e suporte de colocação dos produtos depois do registo

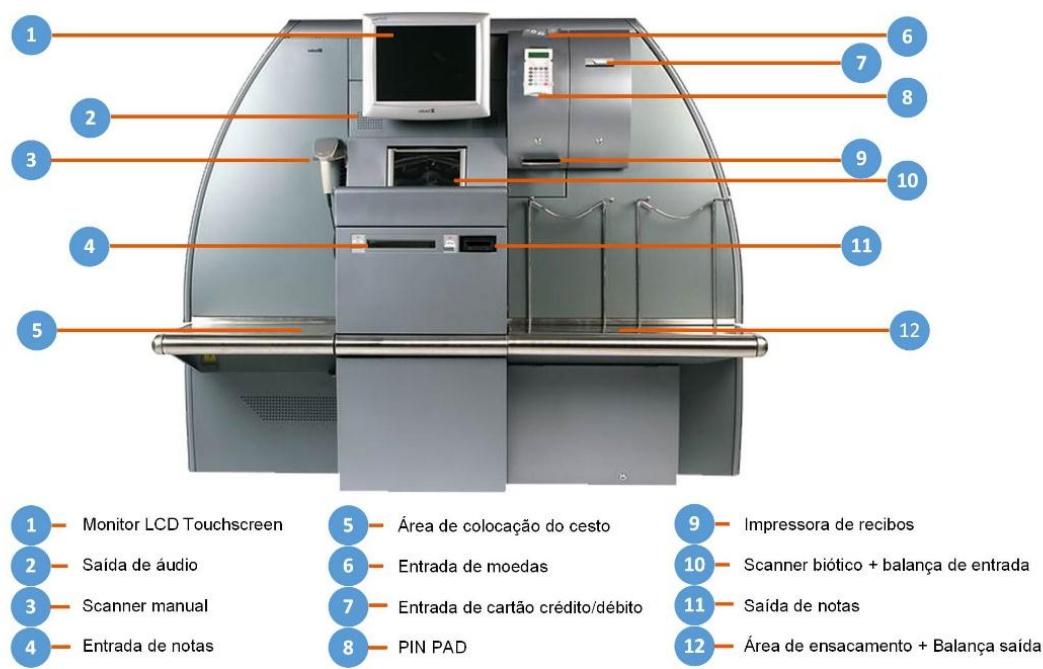

Fig. 2. Periféricos típicos de uma caixa self-checkout. Na figura, máquina Quick Way produzido pela (Adaptado ITAUTEC, 2017)

Estes equipamentos são configurados para um público alvo específico: clientes que têm pouco tempo, que vão frequentemente às compras e que adquirem poucas unidades de cada vez.

Uma das formas de segmentar os clientes à entrada da loja é através da opção pelo utilizador de escolher um cesto (para poucas unidades) ou o carro de compras (para compras de muitas unidades). Outra forma, é impor um limite máximo de unidades nas caixas de self-checkout. A necessidade de colocar os artigos nos sacos e na balança, também limita o tipo de produtos que podem ser comprados, dado que estes devem poder caber nos sacos e nos cesto e em cima da balança de saída. Deste modo, é restingido a utilização da tecnologia a clientes que compram um número reduzido de artigos.

Os equipamentos estão localizados na frente de loja, numa área vedada lateralmente com barras laterais, com um espaço de passagem, uma entrada e uma saída com scanner de segurança. Nesta área, só é permitida a permanência dos clientes durante a utilização do equipamento e do assistente de loja (fig. 3). 
A organização das máquinas na área do checkout pode ser em ilhas no interior deste espaço ou encostadas às zonas laterais. Perto da zona da saída, fica o balcão do assistente que controla entre quatro a seis self-checkouts.

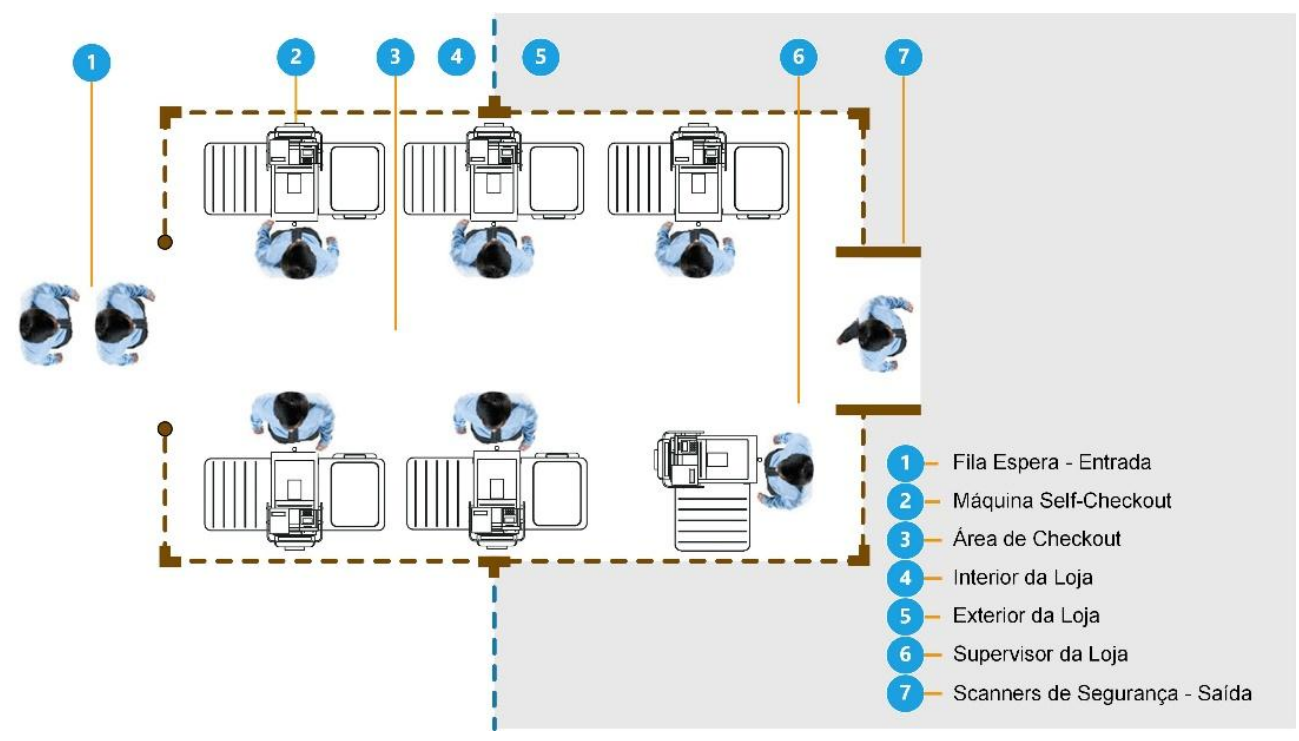

Fig. 3. Disposição típica dos equipamentos numa área de self-checkout.

Os sistemas fornecem um conjunto de funções que permitem aos utilizadores realizar todas as tarefas de uma forma repetitiva e supervisionada, não permitindo que nenhuma modificação seja introduzida por estes.

Os sistemas devem permitir que os utilizadores aprendam bem e realizem o seu trabalho de forma eficiente (Preece, Rogers, Sharp 2005). Para isso, o sistema por um lado deve ser de fácil aprendizagem durante as primeiras utilizações e deve também ser fácil de lembrar como se usa, principalmente nas operações que não se utilizam com muita frequência. Para auxiliar os utilizadores na realização da tarefa, o processo de compra é acompanhado de informações visuais e sonoras, que guiam os clientes através do processo de checkout. Essas informações auxiliam durante as primeiras utilizações a registar os produtos e estimulam a memória, uma vez que, o mesmo processo de registo terá que ser aplicado a todos os produtos e de todas as vezes que a tecnologia for utilizada. No entanto, o utilizador tem de identificar termos para cumprir as instruções, os passos necessários e a sequência correta para a concretização da compra. 
Quando se verificam erros por parte dos clientes, é o próprio sistema que deteta a falha e alerta o assistente que pode esclarecer dúvidas e ajudar a interagir com o sistema.

Das diferentes configurações de equipamentos, os procedimentos base a efetuar por parte do utilizador são similares, compreendidas em três etapas principais (fig 4). Numa primeira etapa, o utilizador regista o produto, que pode ser através da leitura de um código de barras. Depois do produto registado, vai ser ensacado e colocado na balança de saída. Numa terceira, vai ser de efetuado o pagamento em numerário ou em cartão de crédito/débito. $\mathrm{Ou}$ seja, esta tecnologia permite aos consumidores, registar os produtos, empacotar e escolher a forma como pretende pagar os artigos, sem a intervenção do prestador do serviço.

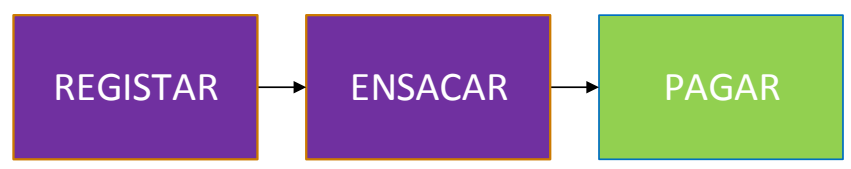

Fig. 4. Etapas principais do procedimento do self-checkout.

O programa de ações da tecnologia poderá ser observada no diagrama de fluxo das ações do utilizador da figura 5 .

O processo inicia-se com a colocação do cesto na balança de entrada. O utilizador dá de seguida a ordem para iniciar a operação de registo e o idioma que pretende nas instruções de voz automáticas no monitor touch-screen. Seguidamente, digitaliza os códigos de barras dos produtos no scanner de leitura óptica, identifica e pesa produtos como frutas e vegetais no monitor touch-screen. Os produtos são depositados um após o outro na balança de saída na área de ensacamento depois de serem registados. Estes são automaticamente validados pelo peso, garantindo a digitalização correta. As informações previamente armazenadas asseguram que o produto correto seja empacotado, permitindo que o cliente prossiga caso os pesos observados e esperados coincidirem. Poderá ainda registar o cartão de cliente, talões de desconto e solicitar que seja emitida uma fatura.

Finalizado o registo, o utilizador efetua o pagamento por um dos vários métodos que são permitidos pelas máquinas como: cartões eletrónicos de crédito e débito ou em notas e moedas.

Qualquer problema que surja durante o processo de registo e pagamento, está presente um assistente pronto a intervir que poderá auxiliar o utilizador a identificar e resolver o problema. Este assistente tem também a função de administrador dado que o seu terminal de supervisão fornece suporte e controlo sobre cada uma das máquinas, podendo bloquear, 
registar produtos e resolver as situações que não estão previstas na configuração para o público.

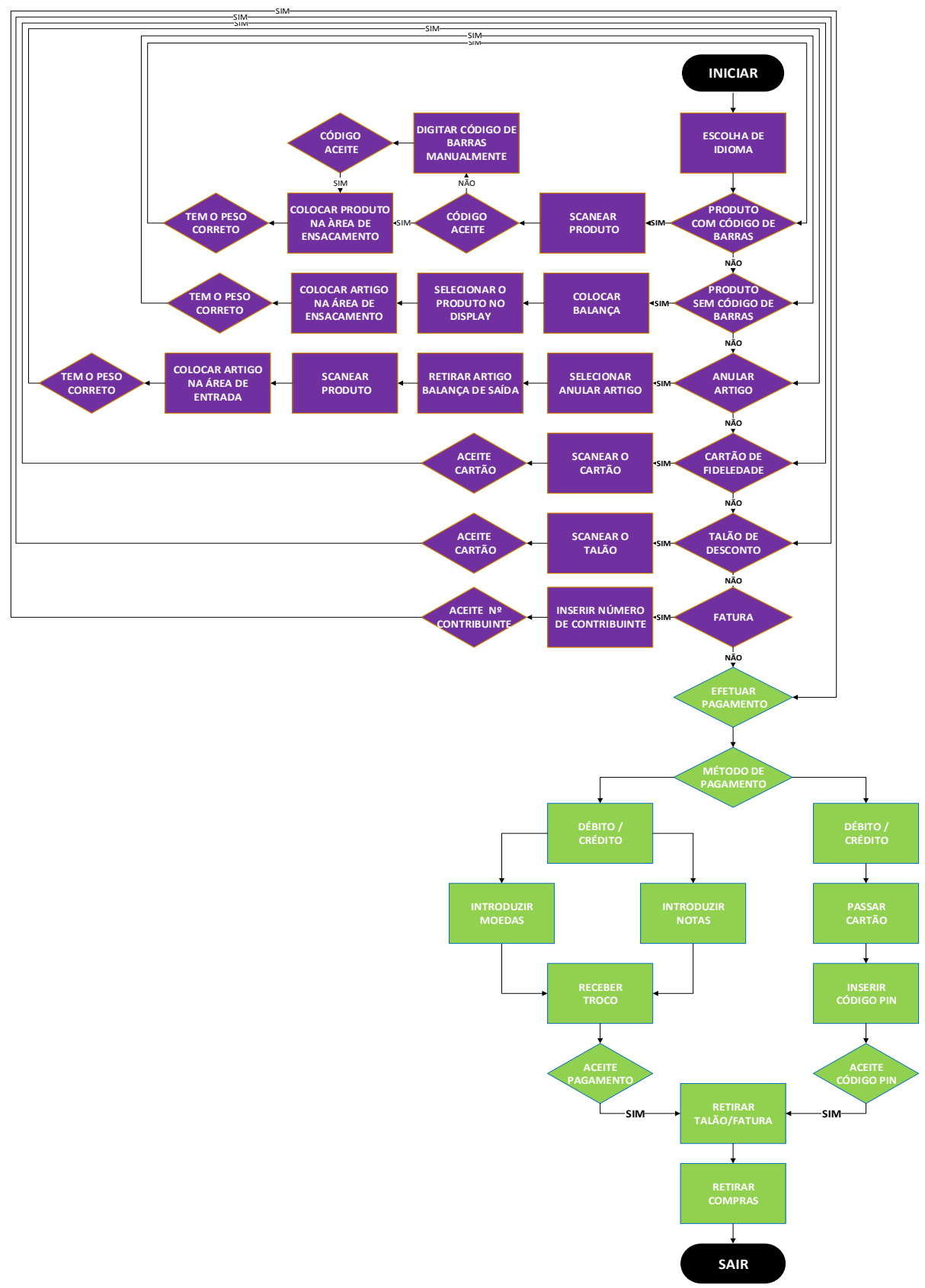

Fig. 5. Diagrama de fluxo das ações do utilizador no self-checkout.

This work is under a $\underline{\text { Creative Commons License CC BY-NC-ND 4.0 }}$ 


\section{Objetivos e Hipóteses}

O principal objetivo desta pesquisa é contribuir para uma melhor compreensão das operações de auto-atendimento em equipamentos self-checkout.

O foco é o estudo da interação homem-sistema através da avaliação percebida dos atributos do sistema durante a sua utilização e do grau de facilidade com que aprendem a usar o equipamento.

Por outro lado, pretende-se também investigar as razões que levam os consumidores a servirem-se das caixas de pagamento automáticas, a avaliação que fazem do serviço e a predisposição que apresentam perante o uso de produtos tecnológicos.

\section{Metodologia}

Foi efetuado um estudo exploratório, descritivo de abordagem quantitativa num estabelecimento retalhista de uma grande superfície comercial, localizada na região centro de Portugal.

Os dados foram recolhidos através de um questionário dirigido aos utilizadores na proximidade das caixas de registo e pagamento automático, self-checkout. Isto permitiu, por um lado, que todos os entrevistados fossem utilizadores da tecnologia em estudo e, por outro, que não tivessem de depender da memória a médio e longo prazo para responder ao inquérito, dada a recente experiência de utilização. $\mathrm{O}$ facto do questionário ter sido feito num único grupo de retalho, fez com que os utilizadores com experiências em diferentes máquinas de self-checkout tivessem pelo menos uma em comum. Neste caso, era a de utilização mais recente.

O questionário aplicado é de autopreenchimento e está dividido em 6 grupos. Os dados foram organizados e analisados através do software Statistic Package for Social Sciences (SPSS 20).

\section{Amostra}

Para o estudo foi definida como população alvo os utilizadores dos equipamentos selfcheckout existentes em grandes superfícies comerciais em Portugal. O efetivo da amostra foi constituído por 400 respostas validadas. 


\section{Resultados}

\subsection{Perfil dos Utilizadores}

Dos resultados obtidos relativos à população da amostra, constata-se que existe uma percentagem mais elevada de utilizadores do sexo feminino $57,5 \%$, enquanto que a do sexo masculino representa $42,5 \%$ (fig. 6).

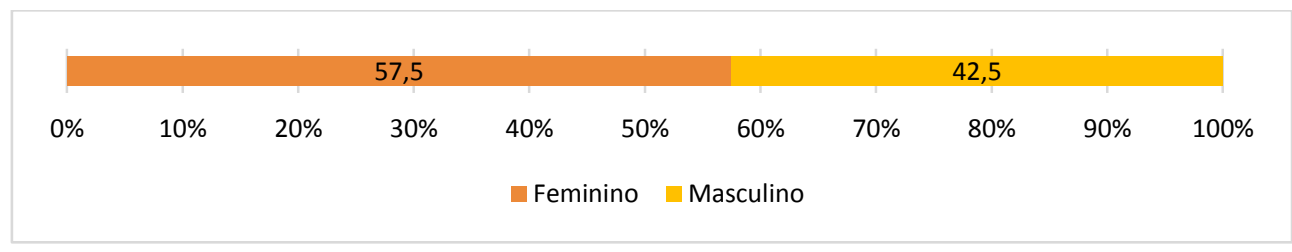

Fig. 6 - Distribuição por sexo dos utilizadores.

Relativamente à distribuição por escalão etário, as diferenças são acentuadas, verificandose que a maior faixa da população tem idades situadas entre os 18-30 anos com 45\%, seguido de uma faixa entre $31-45$ anos com $25,5 \%$ e outra dos $46-60$ anos com 21,8\%. As faixas etárias com menor número de utilizadores correspondem às dos maiores de 60 anos com $6 \%$ e dos menores de 18 anos com 1,8\% (fig.7).

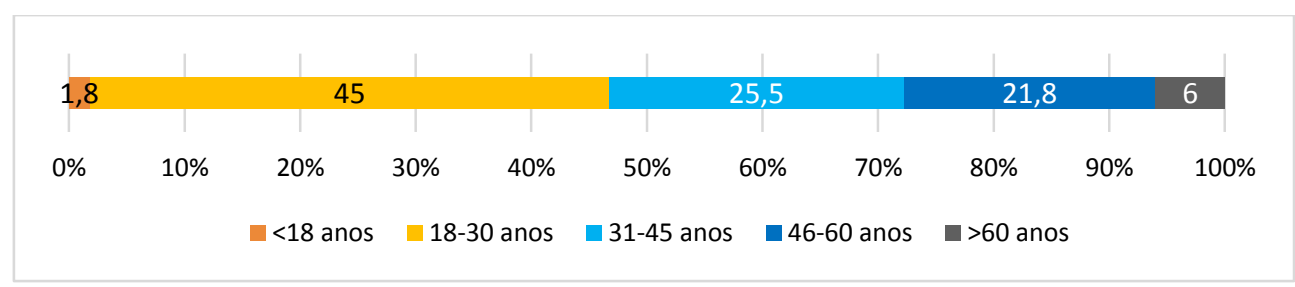

Fig. 7 - Distribuição por grupos etários dos utilizadores

De acordo com os resultados obtidos, mais de metade dos utilizadores tem escolaridade na faixa do $10^{\circ}-12^{\circ}$ anos 54,5\%, seguido dos licenciados $28,8 \%$. Verifica-se ainda, uma distribuição uniforme dos utilizadores com escolaridade entre o $5^{\circ}$ e o $9^{\circ}$ ano $8,5 \%$ e os que têm uma formação superior ao nível do Mestrado ou Doutoramento 8,3\%. A percentagem de utilizadores com grau inferior ao $4^{\circ}$ ano é residual 0,3\% (fig.8). 
Usability and User-Centered Design - User Evaluation Experience in Self-Checkout Technologies

\begin{tabular}{|c|c|c|c|c|c|c|c|c|c|c|}
\hline $0,3 \quad 8,5$ & \multicolumn{6}{|c|}{54,3} & \multicolumn{3}{|c|}{28,8} & 8,3 \\
\hline \multirow[t]{2}{*}{$0 \%$} & $10 \%$ & $20 \%$ & $30 \%$ & $40 \%$ & $50 \%$ & $60 \%$ & $70 \%$ & $80 \%$ & $90 \%$ & $100 \%$ \\
\hline & & 40 ano & 5ㅇ-9으 ano & $\square 1$ & 12 ano & \multicolumn{2}{|c|}{ 口 Licenciatura } & Mest/D & & \\
\hline
\end{tabular}

Fig. 8-Distribuição em função da "Escolaridade” dos utilizadores.

A figura 9 põe em evidência que os utilizadores usam regularmente novas tecnologias como o computador, a internet e os dispositivos móveis, tendo-se obtido respostas de "Sempre" e "Bastante" às questões em cada uma das três categorias, variaram entre os $80,9 \%$ na utilização de Dispositivos Móveis e os 92,2\% no caso de Computador. Pode-se afirmar que a generalidade dos utilizadores entrevistados está habituada a utilizar novas tecnologias.

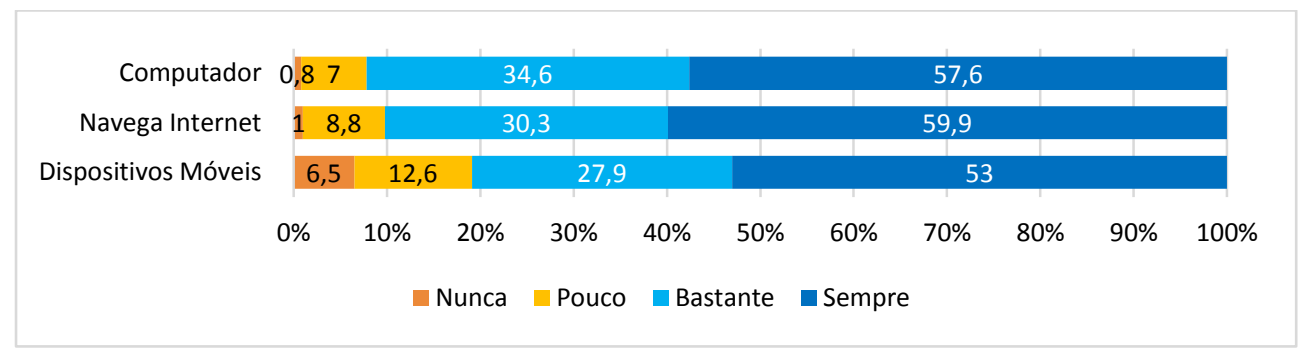

Fig 9 - "Utilização regular de novas tecnologias".

A análise das respostas à pergunta sobre a Frequência de Utilização das caixas de registo e pagamento, verifica-se que 42\% utiliza "Bastante", 33,5\% utiliza "Pouco", 19,3\% utiliza "Muito Pouco" e 4,8\% utiliza "Sempre".

Embora se verifique que os respondentes que utilizam "bastante" as caixas self-checkout tenham estado em maioria, a soma dos que utilizam "pouco" e "muito pouco" totaliza mais de metade das respostas 53,8\% (fig.10). 


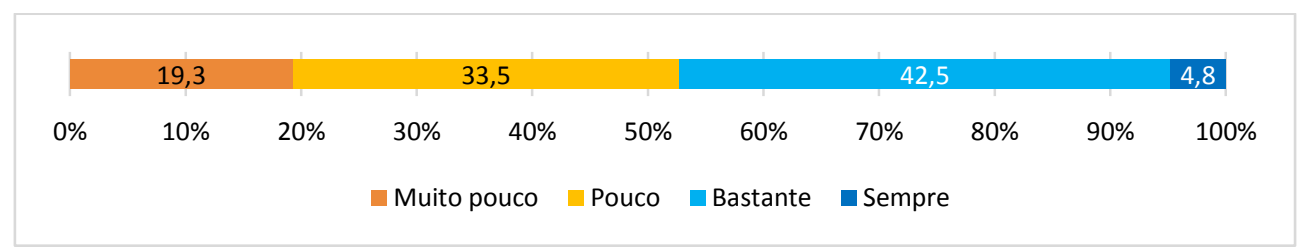

Fig. 10 - "Frequência de Utilização" das caixas de registo e pagamento.

Pretendeu-se saber a variedade de interações em diferentes máquinas de self-checkout. Os resultados demonstram que $44 \%$ utilizou dois sistemas, $28,5 \%$ utilizou um sistema, $17,3 \%$ utilizou três sistemas, $6,5 \%$ utilizou quatro, $2,5 \%$ utilizou cinco e $0,5 \%$ utilizou em mais de cinco (fig.11).

Conclui-se que a maior parte dos utilizadores 73,3\% interagiu com um ou dois sistemas diferentes de registo e pagamento.

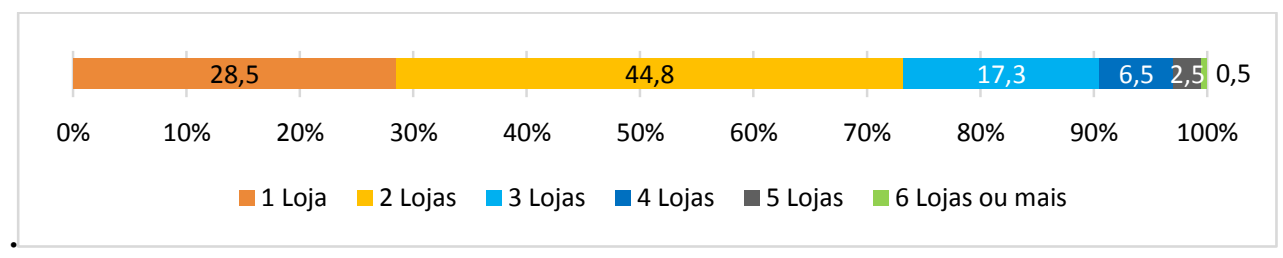

Fig. 11 - Variedade de self-checkouts usados "Número de Lojas em que Interagiram com Sistemas"

\subsection{Grau de Satisfação e Qualidade}

Para verificar se as caraterísticas do produto/serviço correspondem às necessidades do cliente, o questionário contém duas perguntas relativas ao grau de "Satisfação" e de "Qualidade". Estas questões têm a ver com a comparação de desempenho do produto/serviço com as expetativas do utilizador. Os resultados demonstram que a resposta foi positiva às duas questões. Apresentam taxas elevadas de satisfação de $71 \%$ e de qualidade de $76,5 \%$ (fig. 12 ). 


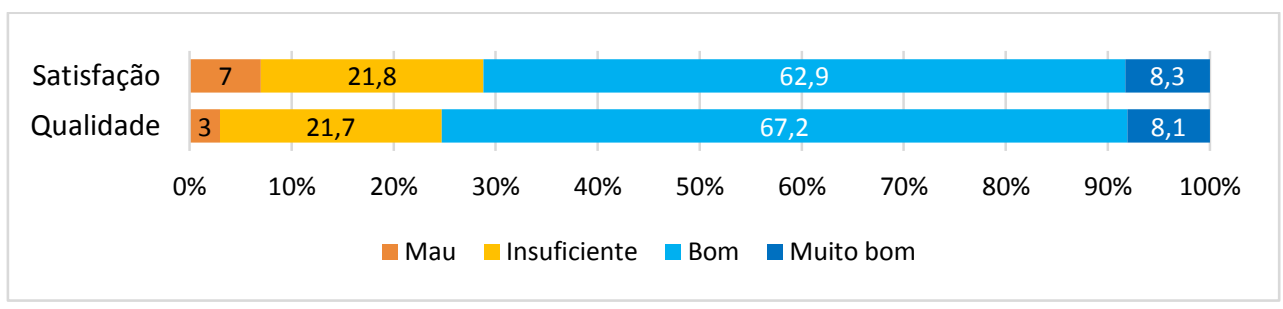

Fig. 12 - Graus de "Satisfação" e de "Qualidade".

Verifica-se ainda que, apesar das taxas de satisfação e qualidade serem altas na pergunta anterior, na questão de âmbito geral sobre se "As caixas self-service são melhores que as caixas tradicionais?", os valores apresentados identificam que apenas $26,6 \%$ dos utilizadores consideram que as caixas self-service são melhores que as caixas com operador (fig.13).

\begin{tabular}{|c|c|c|c|c|c|c|c|c|c|c|}
\hline \multirow[b]{2}{*}{$0 \%$} & \multicolumn{2}{|l|}{20,3} & \multicolumn{4}{|c|}{53,1} & & \multicolumn{2}{|c|}{22,4} & 4,2 \\
\hline & $10 \%$ & $20 \%$ & $30 \%$ & $40 \%$ & 50 & $60 \%$ & $70 \%$ & $80 \%$ & $90 \%$ & $100 \%$ \\
\hline & & scordo & Imente & Dis & & Concordo & Con & do tota & nte & \\
\hline
\end{tabular}

Fig. 13 - "As caixas self-service são melhores que as caixas tradicionais".

\subsection{Grau de Motivação para a Utilização}

Como se pode observar na Figura 14, destacam-se os utilizadores que afirmam que a razão para utilizar o sistema self-checkout decorre de dois aspetos: a existência de grandes "filas de espera nas caixas com operador" $92,6 \%$ e um "volume de compras pequeno" 90,5\%. Outros motivos considerados como importantes são também a "rapidez", a "autonomia" e a "privacidade".

Razões como a "facilidade", "mais controlo", "comodidade", "hábito", "mais eficiente", "prazer" são de menor importância para os utilizadores. É de destacar a má avaliação que obtiveram a eficiência e o prazer, que tiveram avaliações positivas de apenas $26,8 \%$ e $23,9 \%$, respetivamente. 


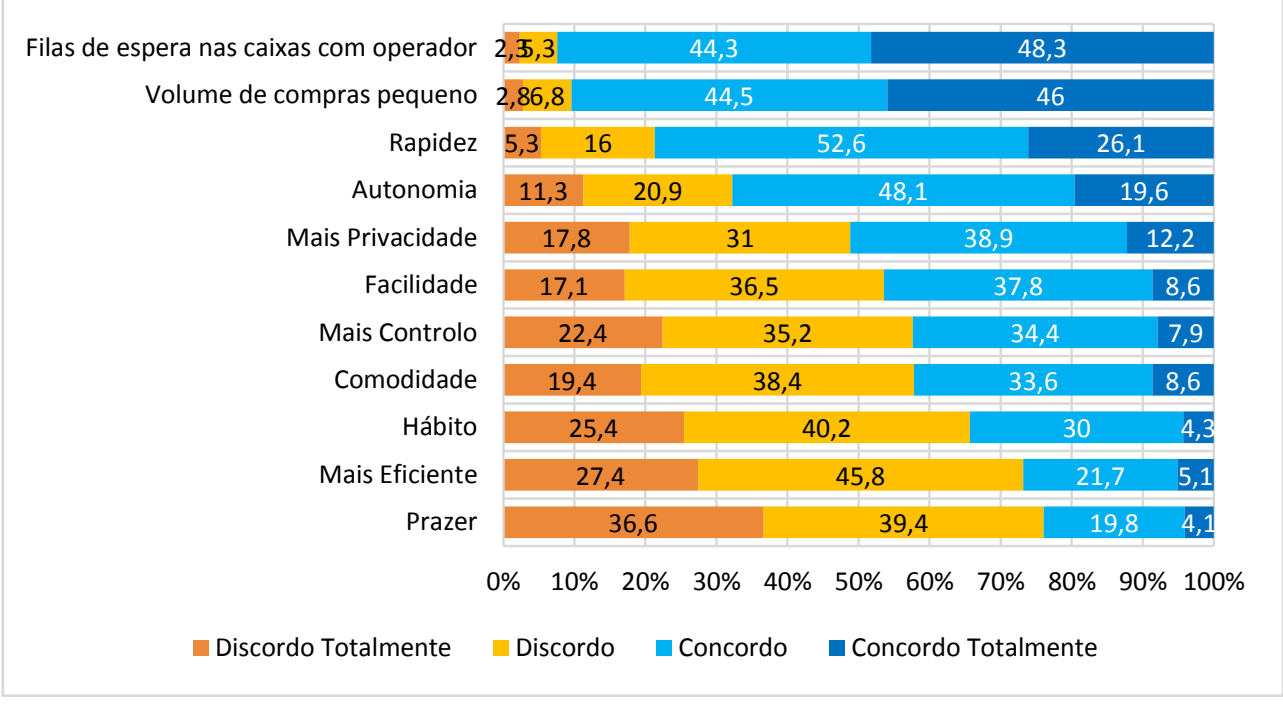

Fig. 14 - "Motivo de utilização dos caixas self-service em vez dos caixas tradicionais".

\subsection{Interação com a Caixa Self-Service}

A maioria dos utilizadores tem a perceção de que domina a tecnologia do serviço de selfcheckout, posto em evidência por $80,6 \%$ dos entrevistados. Os resultados demonstram que $62,8 \%$ dos utilizadores consideram que têm um "Bom" domínio, 17,8\% que têm um Domínio Total (fig.15).

Dos utilizadores que consideram que não dominam o sistema, 16\% afirmam ter "Pouco Domínio" e 3,5\% que "Não Dominam".

\begin{tabular}{|c|c|c|c|c|c|c|c|c|c|}
\hline 3,5 & 16 & & \multicolumn{5}{|c|}{62,8} & \multicolumn{2}{|l|}{17,8} \\
\hline \multirow[t]{2}{*}{$0 \%$} & $10 \%$ & $20 \%$ & $30 \%$ & $50 \%$ & $60 \%$ & $70 \%$ & $80 \%$ & $90 \%$ & $100 \%$ \\
\hline & & & Não domino & Pouco & Bom & Domínio T & & & \\
\hline
\end{tabular}

Fig. 15 - "Domínio das caixas" de registo e pagamento. 
Relativamente à facilidade que os utilizadores tiveram na aprendizagem do uso do sistema, $63,2 \%$ consideraram que a aprendizagem foi "fácil" e 15,8\% que foi "muito fácil", totalizando a sua soma $79 \%$ (fig. 16 ).

Por outro lado, 19\% consideraram que foi "difícil" e $2 \%$ consideraram que foi "muito difícil”.

\begin{tabular}{|c|c|c|c|c|c|c|c|c|c|c|}
\hline 2 & 19 & & \multicolumn{6}{|c|}{63,2} & \multicolumn{2}{|c|}{15,8} \\
\hline \multirow[t]{2}{*}{$0 \%$} & $10 \%$ & $20 \%$ & $30 \%$ & $40 \%$ & $50 \%$ & $60 \%$ & $70 \%$ & $80 \%$ & $90 \%$ & $100 \%$ \\
\hline & & & $\mathrm{Mu}$ & ifícil & Difícil & 口ácil & Muito fác & & & \\
\hline
\end{tabular}

Fig. 16-Grau de "Facilidade em Aprender a Trabalhar com as Caixas Self-checkout".

Para além do domínio e da facilidade de aprendizagem do sistema, 65,3\% dos utilizadores consideram a interação intuitiva. Os resultados demonstram que a "Intuitividade na Interação com Sistema” é positiva (fig.17).

Por outro lado, 34,8\% consideram que o sistema é "pouco" ou "nada" intuitivo.

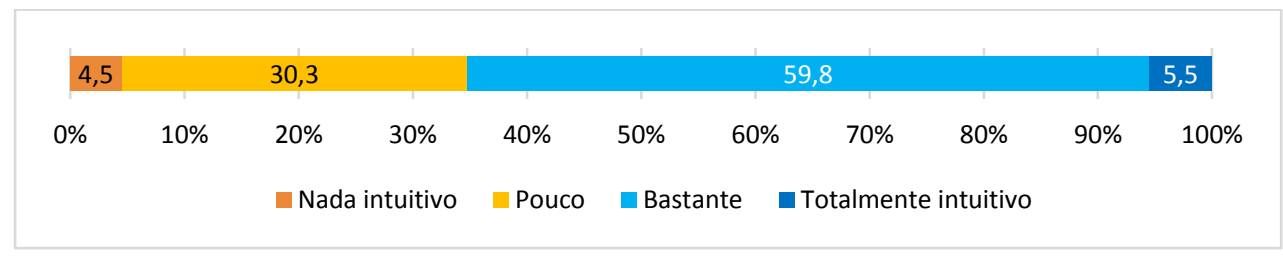

Fig. 17 - Grau de "Intuitividade na Interação com Sistema".

\section{5 . Análise das Variáveis dos Atributos do Sistema}

A caraterização das variáveis dos atributos do sistema self-checkout é apresentada na Figura 18 por ordem crescente das avaliações negativas. Essa organização resulta da soma das avaliações atribuídas de "Muito Mau" e "Negativo".

Num primeiro momento, identificou-se que a grande maioria dos respondentes os classificaram de forma positiva, à exceção de um atributo.

Seguidamente, analizaram-se os atributos com avaliações semelhantes e agruparam-se os resultados em quatro grupos. 
- No $1^{\circ}$ grupo encontra-se um só atributo com avaliação negativa, em que a soma de "Muito Mau" e "Negativo" foi atribuída por $66,7 \%$ dos respondentes. $(25,8 \%+40,9 \%)$

- No $2^{\circ}$ grupo encontram-se os atributos entre $33,4 \%$ e $49,2 \%$. $(8,7 \%+24,7 \%)$ e $(8,1 \%+41,1 \%)$

- No $3^{\circ}$ grupo encontram-se os atributos entre $19,3 \%$ e $27,9 \%$. $(2,9 \%+16,4 \%)$ e $(4,6 \%+23,3 \%)$

- $\mathrm{No}^{\circ}$ grupo encontram-se os atributos entre $7,5 \%$ e $14 \%$. $(1,5 \%+6 \%)$ e $(2,4 \%+11,6 \%)$

Em todos os grupos houve atributos que se destacaram. Os atributos com avaliação mais positiva são os que pertencem ao grupo 4. Os atributos que se consideram críticos do sistema pertencem ao grupo 1 e 2 :

No grupo 1

- "Anular um Artigo Registado" que teve avaliação negativa (66,7\%).

No grupo 2

- "Pesquisa e Registro de Produtos sem Código de Barras" (49,2\%);

- "Registo Manual" de produtos (47,4\%);

- “Área de Ensacamento" (43,9\%);

- "Mensagens de Erro" (42\%);

- "Sensor de Peso" (41,2\%);

- "Pedido de Fatura" (33,4\%).

No grupo 3

- “Área de Colocação do Cesto" 27,9\%;

- "Botões e Grafismos" 26,1\%;

- "Registo do Código de Barras com Scanner Manual" 25,8\%;

- "Registo de Cupões de Desconto" 24,9\%;

- "Localização da Entrada de Notas e Moedas" 24,8\%;

- "Mensagens e Instruções de Voz" 24,6\%;

- "Localização da Saída de Trocos" 23,7\%;

- "Mensagens e Instruções de Texto" 21,3\%;

- "Registo do Cartão de Fidelidade" 20,5\%;

- "Mensagens e Instruções por Imagens" 19,5\%;

- "Registo com Scanner Fixo (código de barras)" 19,3\%. 
No grupo 4

- "Pagamento em Numerário (Notas e Moedas)" (14\%) ;

- "Iniciar o Processo" (13,4\%);

- "Localização do Pin ATM / Multibanco" (13,2\%);

- "Finalização o Registo de Produtos" (10\%);

- "Localização da Ranhura de Recibos/Faturas" (9,9\%);

- "Pagamento com Cartão de Débito/Crédito" (8,1\%);

- "Escolha da Língua (Português, Inglês ..." (7,8\%);

- "Selecionar o Método de Pagamento" (7,5\%).

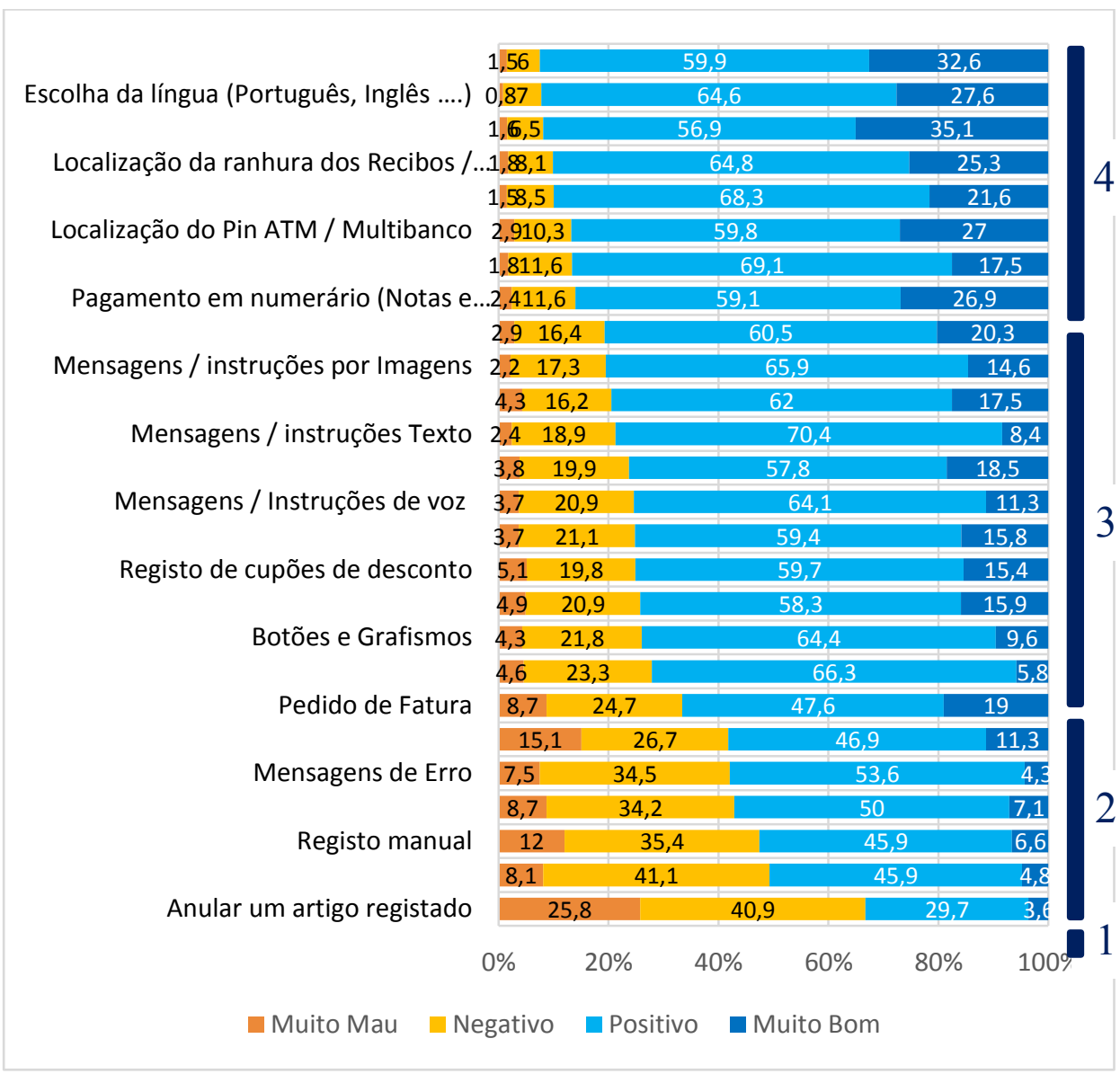

Fig. 18 - "Classificação da Interação com a Caixa Self-Service - Classificação dos atributos do sistema”. 
A figura 19 faz uma análise dos dados da figura 18 separando os atributos de Registo dos de Pagamento.

Os atributos com avaliação mais negativa são dos grupos 1 e 2. Estes pertencem ao processo de registo e podem-se considerar como os atributos críticos do sistema.

Verifica-se ainda que os oito atributos relacionados com o processo de pagamento obtiveram avaliação positiva por parte dos respondentes. Seis encontram-se no grupo 4 e dois no grupo 3 .

Poderá daqui inferir-se que os utilizadores consideram de fácil interação a realização do pagamento na máquina self-checkout. Por outro lado, algumas ações no processo de registo apresentam dificuldades de usabilidade.

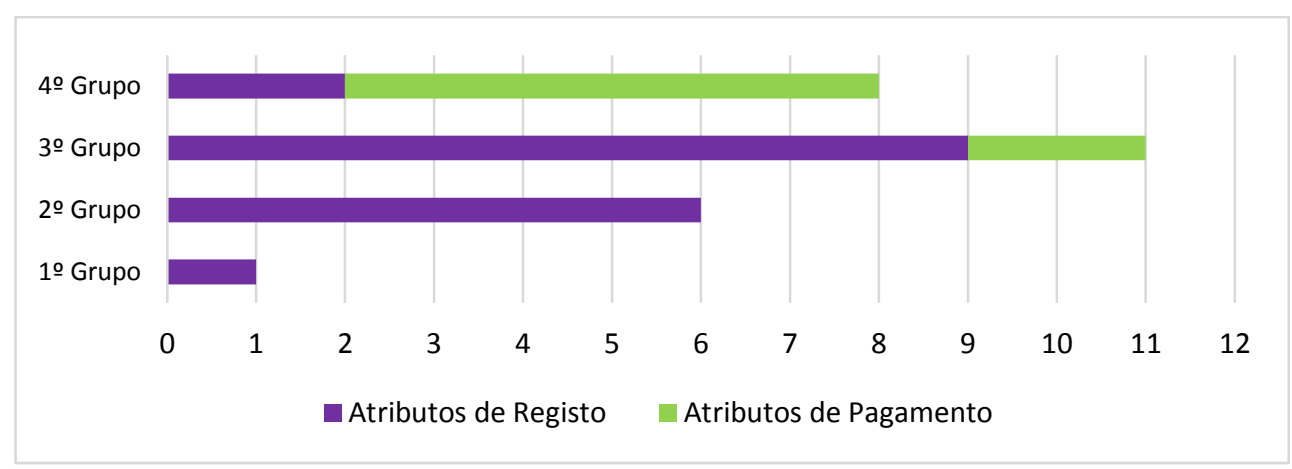

Fig. 19 - Número de atributos de Registo e de Pagamento com avaliações semelhantes.

\section{Discussão}

Com base nos resultados obtidos, é possível inferir a importância atribuída à experiência e avaliação dos utilizadores dos self-checkouts, decorrente da análise das variáveis de interação dos equipamentos eletrónicos no setor do retalho.

A ligação das variáveis satisfação e motivação para o uso e domínio e facilidade de interação, permite determinar o grau de usabilidade dos self-checkouts ligada à importância no design centrado no utilizador e na necessidade dos designers terem conhecimento sobre o desenvolvimento de produtos eletrónicos self-service.

A avaliação isolada de cada atributo do sistema, permite localizar aqueles em que os utilizadores têm menores e maiores dificuldades de interação. 


\section{Conclusões}

O setor do retalho atribui grande importância à implementação das caixas self-checkout, uma vez que responde a uma necessidade cada vez mais premente de contemplar o mercado em geral e os consumidores em particular, com dispositivos self-service que possibilitam a independência do ato de compra do início até ao fim.

Os resultados obtidos com a análise do questionário, revelam que os indivíduos da amostra têm formação escolar média/alta e utilizam com muita regularidade as novas tecnologias. Apresentam um domínio elevado do sistema e consideram que foi fácil a aprendizagem.

As "filas de espera no checkout com operador" e o "volume de compras pequeno", são os principais motivos para a utilização dos equipamentos self-checkout.

O estudo revela ainda um grau elevado de satisfação com o serviço e com a qualidade. Estas variáveis são de elevada importância dado que influenciam a motivação para o uso, que é determinante na experiência de interação. No entanto, em termos comparativos os self-checkout não são considerados melhores que os checkouts com operador.

Com base nos resultados obtidos, os utilizadores percecionam como fácil a interação durante a realização do pagamento. No entanto, embora considerem o mesmo para alguns atributos do registo, existem outros que se apresentam com potenciais dificuldades de interação. A "anulação de artigos registados", a "pesquisa de artigos sem código de barras", o "registo manual", a "área de ensacamento", as "mensagens de erro", o "sensor de peso" e o "pedido de fatura" são sete atributos críticos do sistema.

A importância dada à avaliação por parte do consumidor destes equipamentos, atribui um valor suplementar à necessidade de dar atenção específica ao desenvolvimento de projeto, no sentido de enunciar boas práticas que venham a satisfazer integralmente a experiência de uso dos utilizadores.

A análise da usabilidade orientada para o serviço self-checkout pode ser determinante para a interação utilizador-sistema. As implicações decorrentes das constatações empíricas são discutidas juntamente com orientações para futuras pesquisas.

\section{Referências}

Abrahão, J., et al (2013). Ergonomia e Usabilidade. 1a Edição. São Paulo: Blucher.

Alexandre, J. W. C., et al (2013). Análise do número de categorias da escala de Likert aplicada à gestão pela qualidade total através da teoria da resposta ao item. In: XXIII Encontro Nacional de Engenharia de Produção, Ouro Preto. 
Bettman, J. R., E Park, C. W. (1980), "Effects of Prior Knowledge and Experience and Phase of the Choice Process on Consumer Decision-Processes - a Protocol Analysis", Journal of Consumer Research, Vol. 7, No 3, pp. 234-248.

Castro, D., Atkinson, R., Ezell, J., (2010). Embracing the Self-Service Economy, Information Technology and Innovation Foundation. Available at SSRN: http://dx.doi.org/10.2139/ssrn.1590982

Collier, J.E., \& Kimes, S.E. (2013). Only if it is convenient: Understanding how convenience influences self-service technology evaluation. Journal of Service Research, 16(1), 39-51.

Dabholkar, P.A. (1994), "Incorporating choice into an attitudinal framework: analyzing models of mental comparison processes", Journal of Consumer Research, Vol. 21, pp. 100-18

Dabholkar, P. A. (1996). Consumer Evaluations of New Technology-based Self-service Options: An Investigation of Alternative Models of Service Quality. International Journal of Research in Marketing, Vol. 13, pp. 29-51.

Dabholkar, P.A., Bobbitt, L.M., \& Lee, E.J. (2003). Understanding consumer motivation and behavior related to self-scanning in retailing. International Journal of Service Industry Management, 14(1), 59-95.

Dix, A. et al (2004). Human-Computer Interaction. Third edition. Pearson/Prentice-Hall. New York.

Fernandes, F. et al, (2015). Do Ensaio à Investigação - Textos Breves Sobre a Investigação, Bernabé Hernandis, Carmen Lloret e Francisco Sanmartín (Editores), Oficina de Acción Internacional - Universidade Politécnica de Valência Edições ESAD.cr/IPL, Leiria.

Glazer, N. (1993). Women's Paid and Unpaid Labor. Philadelphia, PA: Temple University Press

Johnson, M. D. (1984), “Consumer Choice Strategies for Comparing Noncomparable Alternatives", Journal of Consumer Research, Vol. 11, No 3, pp. 741-753.

Lee, H., Fairhurst, A., Lee, Min-Young (2009) The Importance of self-service kiosks in developing consumers' retail patronage intentions. Managing Service Quality, 19(6). 687-701.

Litfin, T., \& Wolfram, G. (2010). New Automated Checkout Systems. In Retailing 21st Century, Current and Future Trends (pp. 189-204). Springer

Orel FDL., Kara A. (2014) - Supermarket self-checkout service quality, customer satisfaction, and loyalty: Empirical evidence from an emerging market. Journal of Retailing and Consumer Services, 2014 - Elsevier

Preece, J., Rogers, Y., Sharp, H. (2005) Design de interação. Além da interação homem-computador. Bookmann, Porto Alegre. 